\title{
ON SUBORDINATION OF SUBHARMONIC FUNCTIONS
}

\author{
By Shōjl Kobayashi and Nobuyuki Suita
}

Introduction. In the present paper we are concerned with analytic maps from a Riemann surface into another which preserve the least harmonic majorant of a subharmonic function.

Let $R$ denote an open Riemann surface. Let $S(R)$ be the class of all functions subharmonic on $R$ which admit harmonic majorants on $R$ and $S^{+}(R)=$ $\{f \in S(R): f$ is bounded below on $R\}$. We denote by $\hat{f}$ the least harmonic majorant of $f$ for any $f \in S(R)$. Let $R,(\jmath=1,2)$ be open Riemann surfaces and $\phi$ be an analytic map from $R_{1}$ into $R_{2}$. Littlewood's subordination theorem (see $\left[3\right.$, p. 10]) shows that $f \circ \phi \in S\left(R_{1}\right)$ and

$$
\hat{f} \circ \phi \geqq \hat{f \circ \phi}
$$

on $R_{1}$ for any $f \in S\left(R_{2}\right)$. In this paper we deal with the problem when equality holds in (1).

In the case where $R \in O_{G}$, it is well known that there exist no positive superharmonic functions but the constants on $R$ (see for example [1, p. 204]). Therefore we easily see that $\hat{f}-f \equiv 0$ for any $f \in S(R)$, which means that $S(R)$ reduces to the harmonic functions. It is easily verified that if $R_{1} \subseteq O_{G}$ and $R_{2} \notin O_{G}$ there exist no nonconstant analytic maps from $R_{1}$ into $R_{2}$. Hence, if one of $R_{j}(j=1,2)$ is of class $O_{G}$, equality always holds in (1) for any $f \in S\left(R_{2}\right)$.

From now on we assume that $R_{j} \oplus O_{G}$ for $j=1,2$. Let $G_{j}(z, t)$ denote the Green's function of $R$, with pole at $t$. Following Heins [4], we say that $\phi$ is of type $B 1$ when $G_{2}(\phi(z), t)$ majorates no positive bounded harmonic functions for some $t \in R_{2}$, or equivalently for every $t \in R_{2}$ (see Theorem 4.1 of [4, p. 446]), and we say that $\phi$ is of type $B 1_{1}$ when $G_{2}(\phi(z), t)$ majorates no positive harmonic functions for every $t \in R_{2}$. Let $U$ denote the open unit disc and $\pi$, be a universal covering map of $R_{\jmath}$. By applying the monodromy theorem, we can define an analytic function $\phi$ in $U$ which is bounded by 1 such that

$$
\phi \circ \pi_{1}=\pi_{2} \circ \phi .
$$

An inner function is any function $\phi$ analytic in $U$ with the properties $|\phi(z)| \leqq 1$ in $U$ and $\left|\psi^{*}\left(e^{i \theta}\right)\right|=1$ a. e. on $\partial U$, where $\psi^{*}$ denotes the Fatou's boundary function of $\phi$.

Received May 28, 1979 
1. Main results. First of all we state our results.

THeOREM 1. Let $\phi$ be an analytic map from $R_{1}$ into $R_{2}$, then the following statements are equivalent:

(a) $\hat{f} \circ \phi=\hat{f \circ} \phi$ for every $f \in S^{+}\left(R_{2}\right)$.

(b) $\hat{f} \circ \phi=\hat{f \circ \phi}$ for some $f \in S\left(R_{2}\right)$ whach is not harmonic on whole $R_{\mathrm{u}}$.

(c) There exists an inner function $\phi$ such that $\phi \circ \pi_{1}=\pi_{2}^{\circ} \phi$.

(d) $\phi$ is of type B1.

Remark. Theorem 1 is a generalization of a theorem of Ryff (Theorem 3 of $\left[7\right.$, p. 351]) which states the invariance of $H_{p}$ norm of an analytic function in $U$ under composition by any inner function $\phi$ with $\phi(0)=0$.

TheOrem 2. Let $\phi$ be as in Theorem 1, then the following statements are equivalent:

(a) $\hat{f} \circ \phi=\hat{f \circ \phi}$ for every $f \in S\left(R_{2}\right)$.

(b) There exists an inner function $\phi$ such that $(\psi(z)-\alpha) /(1-\bar{\alpha} \psi(z))$ is a Blaschke product for every $\alpha \in U$ and such that $\phi^{\circ} \pi_{1}=\pi_{2}^{\circ} \phi$.

(c) $\phi$ is of type $B 1_{1}$.

2. Proof of the theorems. First we need a lemma.

Lemma 1. Let $R \notin O_{G}$ and $\pi$ be a unversal coverng map of $R$, then $\hat{f} \circ \pi=$ $\widehat{f \circ \pi}$ holds for any $f \in S(R)$.

Proof. Since $\hat{f} \circ \pi$ is a harmonic majorant of $f \circ \pi$, we easily see that $\hat{f} \circ \pi \geqq$ $\hat{f \circ \pi}$. We must show the inverse inequality. Let $\Gamma$ be the cover transformation group under which $\pi$ is invariant. Since $\widehat{f \circ \pi \circ T}$ is a harmonic majorant of $f \circ \pi \circ T=f \circ \pi$ for every $T \in \Gamma$, we see that $\widehat{\rho} \circ \pi \leqq \hat{f \circ} \pi \circ T$. By composing $T^{-1}$ from

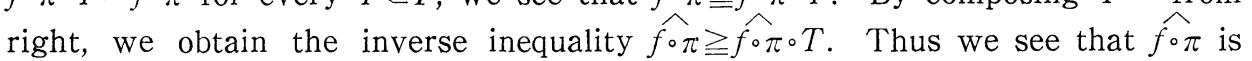
invariant under $\Gamma$. Therefore we can define a single-valued harmonic function on $R$ by $\hat{f \circ \pi} \cdot \pi^{-1}$, which is a harmonic majorant of $f$. Then we see that

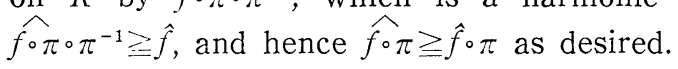

Remark. Lemma 1 was essentially proved by Rudin [5, p. 48].

Proof of Theorem 1.

1. (a) implies (d). Suppose that (d) does not hold. Then there exists a positive bounded harmonic function $u$ which is majorated by $G_{2}(\phi(z), t)$ on $R_{1}$ for some $t \in R_{2}$. Let $g(z)=-\min \left\{G_{2}(z, t), M\right\}$, where $M=\sup \left\{u(z): z \in R_{1}\right\}$, then $g \in S^{+}\left(R_{2}\right)$ and $\hat{g \circ} \phi \leqq-u$. On the other hand, we see by (a) that $\widehat{g \circ} \phi=\hat{g}^{\circ} \phi \equiv 0$, since $\hat{g} \geqq-\hat{G}_{2} \equiv 0$. This is a contradiction. 
2. (d) implies (b). For example, let $f=-\min \left\{G_{2}(z, t), 1\right\}$ for some $t \in R_{2}$, then we see by (d) that $\widehat{f \circ \phi} \equiv 0 \equiv \hat{f} \circ \phi$.

3. (b) implies (c). Suppose that (c) does not hold, then every analytic function $\phi$ in $U$ bounded by 1 such that $\phi \circ \pi_{1}=\pi_{2} \circ \psi$ is not inner. Therefore the set $E=\left\{e^{2 \theta} \cdot\left|\phi^{*}\left(e^{2 \theta}\right)\right| \leqq 1-2 \delta\right\}$ is of positive measure for a sufficiently small positive number $\delta$. By Egorov's theorem we can find a compact subset $F$ of $E$ of positive measure on which $\phi\left(r e^{i \theta}\right)$ converges uniformly to $\psi^{*}\left(e^{i \theta}\right)$ as $r \rightarrow 1$. Then, we have $\left|\psi\left(r e^{2 \theta}\right)\right| \leqq 1-\delta$ for every $e^{\imath \theta} \in F$ and every $r \geqq r_{0}$ for some $r_{0}$ with $0<r_{0}<1$. Let $f$ be as in (b), then we see that $\hat{f}>f$ on $R_{2}$, since $f$ is not harmonic on $R_{2}$. Let $\varepsilon=\inf \left\{\hat{f}(\zeta)-f(\zeta): \zeta \in \pi_{2}(\{|z| \leqq 1-\delta\})\right\}>0$, then we see that

$$
\left(\hat{f} \circ \pi_{2} \circ \phi\right)\left(r e^{\imath \theta}\right) \geqq\left(f \circ \pi_{2} \circ \phi\right)\left(r e^{\imath \theta}\right)+\varepsilon
$$

if $r \geqq r_{0}$ and $e^{\imath \theta} \in F$. Then, by (3), we see for $r \geqq r_{0}$

$$
\begin{aligned}
& \left(\hat{f} \circ \phi \circ \pi_{1}\right)(0)=\left(\hat{f} \circ \pi_{2} \circ \phi\right)(0) \\
& =\frac{1}{2 \pi} \int_{0}^{2 \pi}\left(\hat{f}_{\circ} \pi_{2^{\circ}} \circ\right)\left(r e^{\imath \theta}\right) d \theta \\
& =\frac{1}{2 \pi}\left(\int_{F}+\int_{F c}\left(\hat{f} \circ \pi_{2} \circ \psi\right)\left(r e^{\imath \theta}\right) d \theta\right) \\
& \geqq \frac{1}{2 \pi}\left(\int_{F}\left(f \circ \pi_{2}^{\circ} \phi\right)\left(r e^{\imath \theta}\right)+\varepsilon\right) d \theta \\
& \left.+\int_{F^{c}}\left(f \circ \pi_{2} \circ \psi\right)\left(r e^{\imath \theta}\right) d \theta\right) \\
& =\frac{1}{2 \pi} \int_{0}^{2 \pi}\left(f \circ \pi_{2}{ }^{\circ} \psi\right)\left(r e^{\imath \theta}\right) d \theta+\varepsilon m(F),
\end{aligned}
$$

where $m$ denotes the normalized Lebesgue measure. Letting $r \rightarrow 1$, we see $\left(\hat{f} \circ \phi \circ \pi_{1}\right)(0)>\left(\hat{f} \circ \pi_{2} \circ \phi\right)(0)$. Then, using Lemma 1, we obtain $\hat{f} \circ \phi>\hat{f} \circ \phi$, as desired.

4. (c) implies (a). Let $f \in S^{+}\left(R_{2}\right)$ and we assume that (c) holds. Without loss of generality, we may assume that $f$ is nonnegative on $R_{2}$. Let $\rho$ be fixed with $0<\rho<1$ and let $M_{o}=\sup \left\{\left(f \circ \pi_{2}\right)(z):|z| \leqq \rho\right\}$. By Egorov's theorem, for every $\varepsilon>0$, there exists an open subset $O$ of the unit circle such that

$$
m(O)<\varepsilon / M_{\rho}
$$

and that $\dot{\varphi}\left(r e^{i \theta}\right)$ converges uniformly to $\psi^{*}\left(e^{i \prime}\right)$ on $O^{c}$. Therefore there exists $r$ with $0<r<1$ such that

$$
\left|\phi\left(r e^{i \theta}\right)\right|>\rho
$$

for $e^{2 \theta} \in O$, since $\left|\psi^{*}\left(e^{2 \theta}\right)\right|=1$ a. e. on $\partial U$. Let $u_{o}$ be the least harmonic majorant of $f \circ \pi_{2}$ in $\Delta_{\rho}=\{z:|z|<\rho\}$. Let $D_{\rho}=\phi^{-1}\left(\Delta_{\rho}\right)$ and $\Omega_{\rho}$ be the connected component of $D_{o} \cap \Delta_{r}$ containing 0 . Then, by (6), we see that 


$$
\partial \Omega_{\rho} \cap \Gamma_{r} \subset r O \equiv\left\{r e^{i \theta}: e^{i \theta} \in O\right\}
$$

Let $\omega$ be the harmonic measure of $r O$ in $\Delta_{r}$, then by (5)

$$
w(0) \leqq \varepsilon / M_{\rho} .
$$

Let $h_{\rho}=\widehat{f \circ \pi_{2} \circ \phi}-u_{\rho} \circ \phi$, then we easily see that

$$
h_{\rho} \geqq 0 \quad \text { on } \partial \Omega_{\rho}-\partial \Delta_{r},
$$

and

$$
h_{\rho} \geqq-M_{\rho} \text { on } \partial \Omega_{\rho} \cap \partial \Delta_{r} .
$$

Therefore, by the maximum principle, we obtain

$$
h_{\rho} \geqq-M_{\rho} \omega \text { in } \Omega_{\rho},
$$

and hence $h_{\rho}(0) \geqq-\varepsilon$. Since $\varepsilon$ is arbitrary, we see that $h_{\rho}(0) \geqq 0$. Letting $\rho \rightarrow 1$, we obtain

$$
\widehat{f \circ \pi_{2} \circ \phi} \geqq \widehat{f \circ \pi_{2} \circ \psi}
$$

since $\lim _{\rho \rightarrow 1} u_{\rho}=\hat{f \circ \pi_{2}}$. Using Lemma 1 , we obtain $\hat{f \circ \phi} \geqq \hat{f} \circ \phi$, as desired.

Proof of Theorem 2.

1. (a) implies (b). Suppose that (b) does not hold, then there exists $\alpha \in U$ such that $(\psi(z)-\alpha) /(1-\bar{\alpha} \psi(z))$ is not a Blaschke product. Let $S$ denote its singular part, then $S$ is represented as

$$
S(z)=\exp \left(-\int_{0}^{2 \pi} \frac{e^{i \theta}+z}{e^{i \theta}-z} d \mu(\theta)\right)
$$

where $\mu$ is a positive singular measure on $\partial U$ (see $[3$, p. 24]). Let $f(z)=$ $-G_{2}\left(z, \pi_{2}(\alpha)\right)$, then $f \in S\left(R_{2}\right)$ and

$$
\left(f \circ \pi_{2}\right)(z)=\sum_{T \in \Gamma} \log \left|\begin{array}{c}
T(z)-\alpha \\
1-\bar{\alpha} T(z)
\end{array}\right|
$$

by Myrberg's theorem (see [8, p. 522]). Therefore

$$
\begin{aligned}
\left(f \circ \phi^{\circ} \pi_{1}\right)(z) & =\left(f \circ \pi_{2} \circ \phi\right)(z) \\
& =\sum_{T \in \Gamma} \log \left|\frac{(T \circ \psi)(z)-\alpha}{1-\bar{\alpha}(T \psi)(z)}\right| \\
& =\log \left|\frac{\phi(z)-\alpha}{1-\bar{\alpha} \psi(z)}\right|+\sum_{\substack{T \in \Gamma^{J} \\
T \neq 2 d .}} \log \left|\begin{array}{c}
(T \circ \phi)(z)-\alpha \\
1-\bar{\alpha}(T \circ \psi)(z)
\end{array}\right| \\
& \leqq \log |S(z)| \\
& =-\int_{0}^{2 \pi} \operatorname{Re} \frac{e^{i \theta}+z}{e^{i \theta}-z} d \mu(\theta),
\end{aligned}
$$


and hence

$$
f \circ \phi \circ \pi_{1} \leqq-P[\mu]<0,
$$

where $P[\mu]$ denotes the Poisson integral of $\mu$. Using Lemma 1, we have $\hat{f \circ \phi}<\hat{f} \circ \phi$ from which we see that (a) does not hold.

2. (b) implies (c). The following lemma is well known, for the proof, for example see $[6$, p. 335$]$.

LEMma 2. Let $B$ be a Blaschke product, then

$$
\lim _{r \rightarrow 1} \frac{1}{2 \pi} \int_{0}^{2 \pi} \log \left|B\left(r e^{i \theta}\right)\right| d \theta=0 .
$$

Remark. (17) means

$$
\widehat{\log |B|} \equiv 0
$$

in our language, since the left-hand side of (17) is the value at 0 of the least harmonic majorant of $\log |B|$.

Let $f(z)=-G_{2}(z, t)$ for arbitrarily fixed $t \in R_{2}$. Then, by Myrberg's theorem,

$$
\left(f \circ \pi_{2}\right)(z)=\sum_{T \in \Gamma} \log \left|\frac{T(z)-\alpha}{1-\bar{\alpha} T(z)}\right|,
$$

where $\alpha$ is a point in $U$ with $\pi_{2}(\alpha)=t$. Therefore we see

$$
\begin{aligned}
\left(f \circ \phi \circ \pi_{1}\right)(z) & =\left(f \circ \pi_{2} \circ \phi\right)(z) \\
& =\sum_{T \in \Gamma} \log \left|\frac{(T \circ \phi)(z)-\alpha}{1-\bar{\alpha}(T \circ \phi)(z)}\right| \\
& =\sum_{T \in \Gamma} \log \left|B_{T}(z)\right| \\
& =\log |B(z)|,
\end{aligned}
$$

where $B_{T}=(T \circ \psi-\alpha) /(1-\bar{\alpha} T \circ \psi)$, which is a Blaschke product by (b) for every $T \in \Gamma$, and $B=\prod_{T \in \Gamma} B_{T}$. Using Lemma 2, we obtain

$$
\widehat{f \circ \phi^{\circ} \pi_{1}}=\widehat{\log |B|} \equiv 0 .
$$

Then, by Lemma 1 , we see that $\widehat{f \circ \phi} \equiv 0$, which means that $\phi$ is of type $B 1_{1}$, as desired.

3. (c) implies (a). Any superharmonic function $s$ on a Riemann surface $R \notin O_{G}$ which is represented as

$$
s(z)=\int_{R} G(z, t) d \mu(t),
$$

where $G$ is the Green's function of $R$ and $\mu$ is a nonnegative measure on $R$, is called a (Green's) potential. By Riesz's theorem (Satz 4.6 and Folgesatz 4.6 of 
[2, pp. 41-42]), a nonnegative superharmonic function is a potential if and only if its greatest harmonic minorant is 0 . Let $f \in S\left(R_{2}\right)$, then $\hat{f}-f$ is a potential on $R_{2}$, i. e.

$$
\hat{f}(w)-f(w)=\int_{R_{2}} G_{2}(w, \zeta) d m(\zeta),
$$

where $m$ is a nonnegative measure on $R_{2}$. By (c) $G_{2}(\phi(z), t)$ is a potential on $R_{1}$ for any $t \in R_{2}$, i. e.

$$
G_{2}(\phi(z), t)=\int_{R_{1}} G_{1}(z, \tau) d \nu_{t}(\tau)
$$

where $\nu_{t}$ is a nonnegative measure on $R_{1}$ for every $t \in R_{2}$. It 1 s known that $\nu_{t}$ is the sum of the point masses at points $s$ such that $\phi(s)=t$ counting with multiplicity (see [4, p. 440]). Therefore we can easily see that for any compact $K \subset R_{1}, \nu_{t}(K)$ is upper semi-continuous as a function of $t$. Define a nonnegative measure $\nu$ on $R_{2}$ by

$$
\nu(K)=\int_{R_{2}} \nu_{t}(K) d m(t),
$$

for any compact $K \subset R_{1}$. From (23), (24) and (25) we obtain

$$
(\hat{f} \circ \phi)(z)-(f \circ \phi)(z)=\int_{R_{1}} G_{1}(z, \tau) d \nu(\tau),
$$

which means that $\hat{f} \circ \phi-f \circ \phi$ is a potential on $R_{1}$, and hence its greatest harmonic minorant is 0 by Riesz's theorem cited above (cf. [4, pp. 449-451]). Therefore we obtain $\hat{f} \circ \phi=\widehat{f \circ \phi}$, as desired.

\section{REFERENCES}

[1] Ahlfors, L.V. Axd L. Sario, Riemann surfaces, Princeton university press, 1960.

[2] Constantinescu, C. And A. Cornea, Ideale Ränder Riemannscher Flächen, Springer-Verlag, 1963.

[3] Duren, P.L. Theory of $H^{p}$ spaces, Academic Press, New York, 1970.

[4] Heins, M. On the Lindelöf principle, Ann. of Math. 61 (1955), 440-473.

[5] Rudin, W. Analytic functions of class $H_{p}$, Trans. Amer. Math. Soc. 78 (1955), 46-66.

[6] Rudin, W. Real and complex analysis, MacGraw-Hill, New York, 1974.

[7] RyfF, J.V. Subordinate $H^{p}$ functions, Duke Math. J. 33 (1966), 347-354.

[8] Tsuji, M. Potential theory in modern function theory, Maruzen, Tokyo, 1959.

Department of Mathematics,

Tokyo Institute of Technology. 